\title{
Un camino hacia el cambio en el aprendizaje: La expedición como recurso pedagógico en el ámbito universitario'
}

\author{
A Way to Change Learning: Field Trips as a University Pedagogical Resource
}

\author{
Érika Vásquez Salazar ${ }^{2}$ \\ Universidad Nacional \\ División de Educación Básica,Centro de Investigación y Docencia en Educación \\ Heredia, Costa Rica \\ erika.vasquez.salazar@una.cr
}

\begin{abstract}
Recibido 30 de abril de 2013 • Corregido 5 de agosto de 2013 • Aceptado 11 de setiembre de 2013
Resumen. Este artículo representa la sistematización de diferentes experiencias con expediciones pedagógicas en educación superior, las cuales se desarrollaron entre los años 2005 y 2012 en la División de Educación Básica (DEB) en la Universidad Nacional. La expedición fue utilizada como un recurso pedagógico para el desarrollo de actitudes y aptitudes con estudiantes de varias carreras de educación. La autora señala la expedición pedagógica como una de las posibles estrategias que se pueden utilizar para promover la innovación en la educación superior y lograr un cambio positivo en el abordaje de los procesos de enseñanza y aprendizaje. Se presenta la expedición como una herramienta que le brinda al estudiantado una oportunidad que le permite concretar su plan de estudios de una forma más significativa, participativamente y en contacto con la realidad educativa; además de que le humaniza y le ayuda a reinventarse como persona y como futuro docente.
\end{abstract}

Palabras claves. Expedición pedagógica, gira, educación superior, formador de formadores.

Abstract. This article represents the systematization of different experiences related to educational field trips in higher education taking place between 2005 and 2012 within the Primary Education Division (DEB) at Universidad Nacional, Costa Rica. Field trips were used as an educational resource to develop attitudes and aptitudes in students from various education majors. The author proposes pedagogical field trips as a possible strategy to promote innovation in higher education and achieve a positive change in teaching and learning processes. Field trips are presented as a tool that allows students to complete their coursework in a more significant and participative manner being in contact with the educational reality and to become humanized and to reinvent themselves as persons and future teachers.

Keywords. Educational field trips, higher education, trainer of trainers.

1 Todas las fotografías que ilustran el texto son propiedad de la autora y fueron tomadas durante las diferentes expediciones pedagógicas realizadas. Talamanca, 2007.

2 Académica en la División de Educación Básica del Centro de Investigación y Docencia en Educación de la Universidad Nacional, Costa Rica. Máster con especialidad en Pedagogía de la Universidad Nacional. Bachiller en Ciencias de la Educación ly ll ciclo concentración Inglés de la Universidad Estatal a Distancia. Licenciada en Docencia de la Universidad Estatal a Distancia. Actualmente es gestora de la carrera de Enseñanza del Inglés para I y ll Ciclos. 
URL: http://www.una.ac.cr/educare

CORREO: educare@una.cr

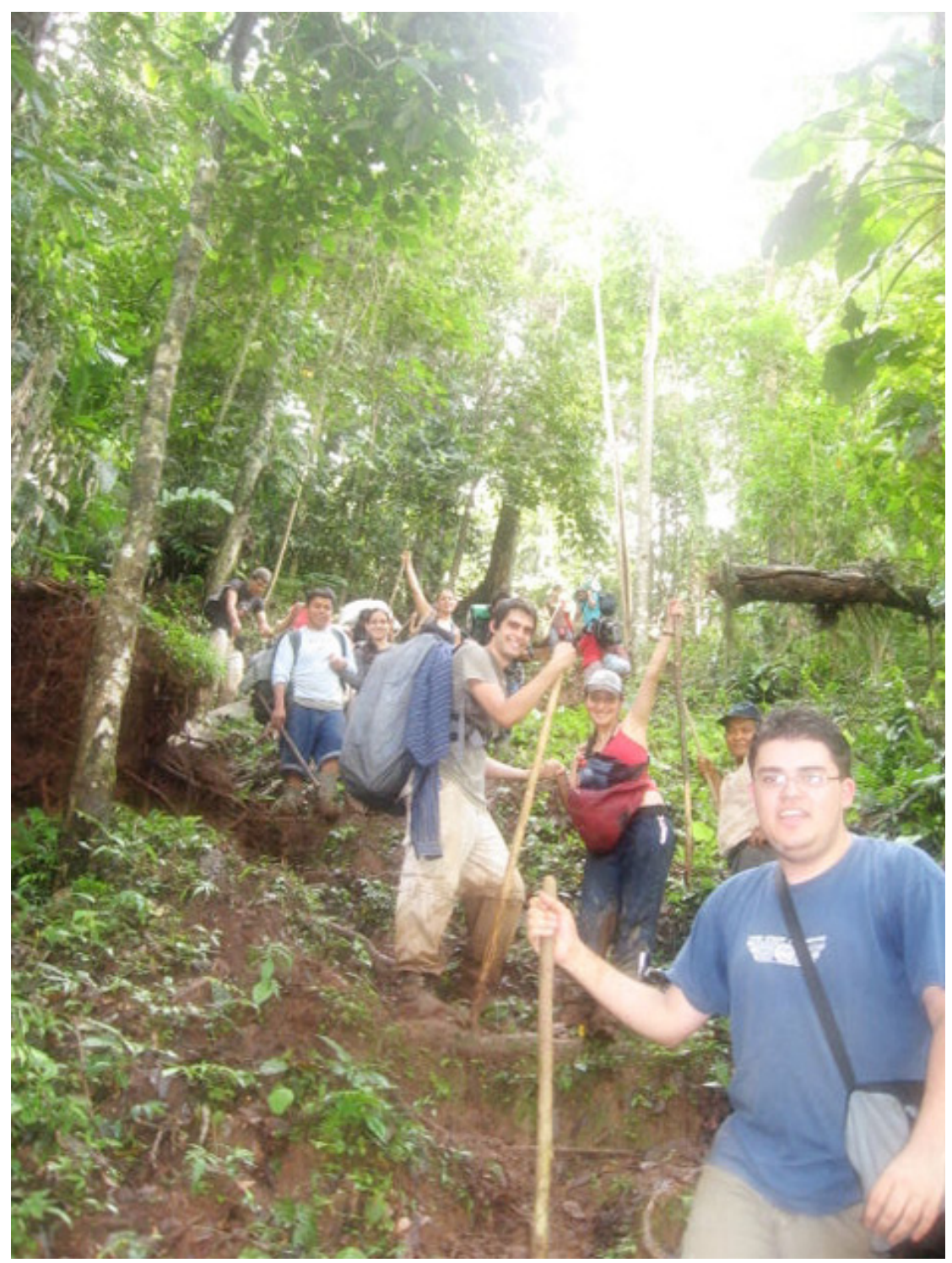

Figura 1. Expedición pedagógica. Talamanca 2007.

La Expedición Pedagógica es un viaje. En ella somos viajeros, somos [sic] Expedicionarios, pero en esencia somos maestros con capacidad de reflexionar y de recibir los nuevos vientos que surcan los aires y traen con ellos experiencias, movimiento, cambio, apertura y asombro. (Adalgiza et al., 2005, p. 4)

Ver figura 1 


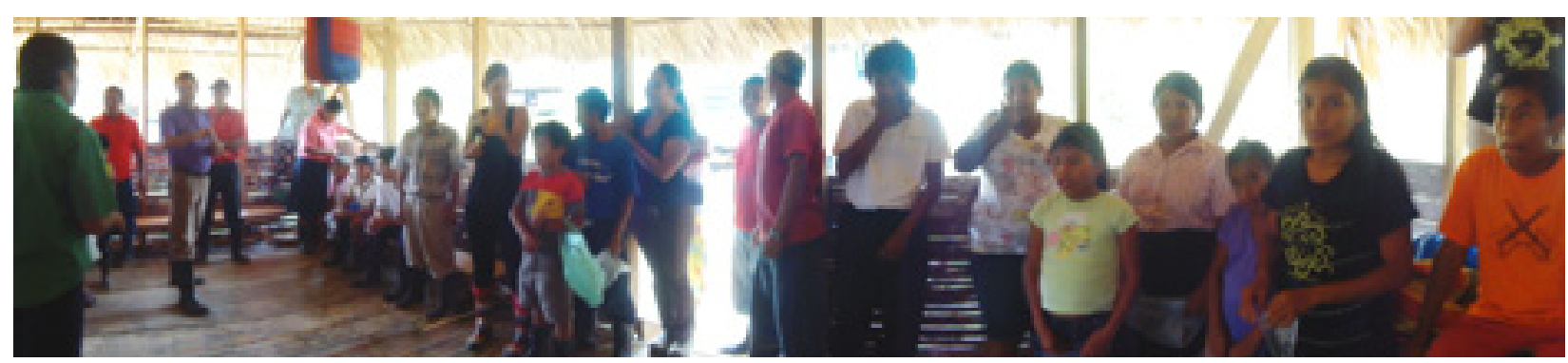

Figura 2. Reunión con la comunidad de Alto Urén, Talamanca, 2011.

El contexto universitario se circunscribió, por mucho tiempo, al espacio áulico, dejando de lado muchos otros escenarios que ofrecen opciones muy variadas para la formación del estudiantado, las cuales correctamente implementadas pueden ayudar a lograr el éxito en la educación superior, en ese sentido, el Ministerio de Educación de Ciencia y Tecnología [MECT] y la Organización de los Estados Americanos, Agencia Internacional para la Cooperación y el Desarrollo [OEA-AICD] (2005), afirman que:

De lo que se trata, entonces, es de ensayar y poner a prueba otros modos de formarnos, de transformarnos, sin anticipar caminos seguros a prueba de desgracias escolares, sino abriendo paso a un sendero sinuoso y estimulante, colmado de desafíos y de preguntas, que pongan en posición de pensar qué les pasa a los docentes con esto que pasa en la formación y en las escuelas, sin que ello implique resignarse a lo que hay o renunciar a producir otros efectos de sentido en las trayectorias de formación y de desarrollo profesional de los docentes. La intención es abrir, redefinir, recrear y maximizar el trabajo pedagógico que tiene encomendado el abanico complejo, heterogéneo y singular que presentan las instituciones formadoras de docentes. (p. 14)

De acuerdo con lo anterior, en los formadores de formadores recae la responsabilidad de abrir caminos que lleven al estudiantado por horizontes antes no explorados. Se debe repensar el acto educativo y llevar más allá del tradicionalismo -que hoy se ha tratado de renovar mediante las tecnologías y otras metodologías, pero se ha olvidado de incluir el contexto y la realidad del mundo que nos rodea-. Es imperante el poder brindar las herramientas al estudiantado para que pueda mirar el mundo de una manera diferente, y que lo pueda transformar por medio de su relación con el entorno (Ver figura 2).

En ese sentido Torío, Peña y Fernández (2010, p. 249) comparten que se debe "formar a un estudiante activo mediante el desarrollo de una actitud participativa y no meramente pasiva y receptiva, para que pueda comprender y enjuiciar con más profundidad la materia que estudia". Lo anterior, enfatiza diferentes características deseables en el alumnado, tales como participación y capacidad crítica y surge la interrogante de cómo lograr desarrollar estas 
y otras actitudes a través de los diferentes cursos que se imparten en un plan de estudios (ver figura 3), y que contribuyen a la búsqueda de la calidad, entendiéndola, tal como lo cita Muñoz (2005), como: "la calidad no se reduce a un criterio de eficiencia cuantificable, sino que abarca la profundidad del compromiso humano hacia el presente y el futuro de todas las personas" (p. 27), sobre todo si se habla de la formación de personal formador, el cual, posteriormente, será el encargado de guiar los procesos de enseñanza y aprendizaje de las nuevas generaciones. El mismo autor señala que:

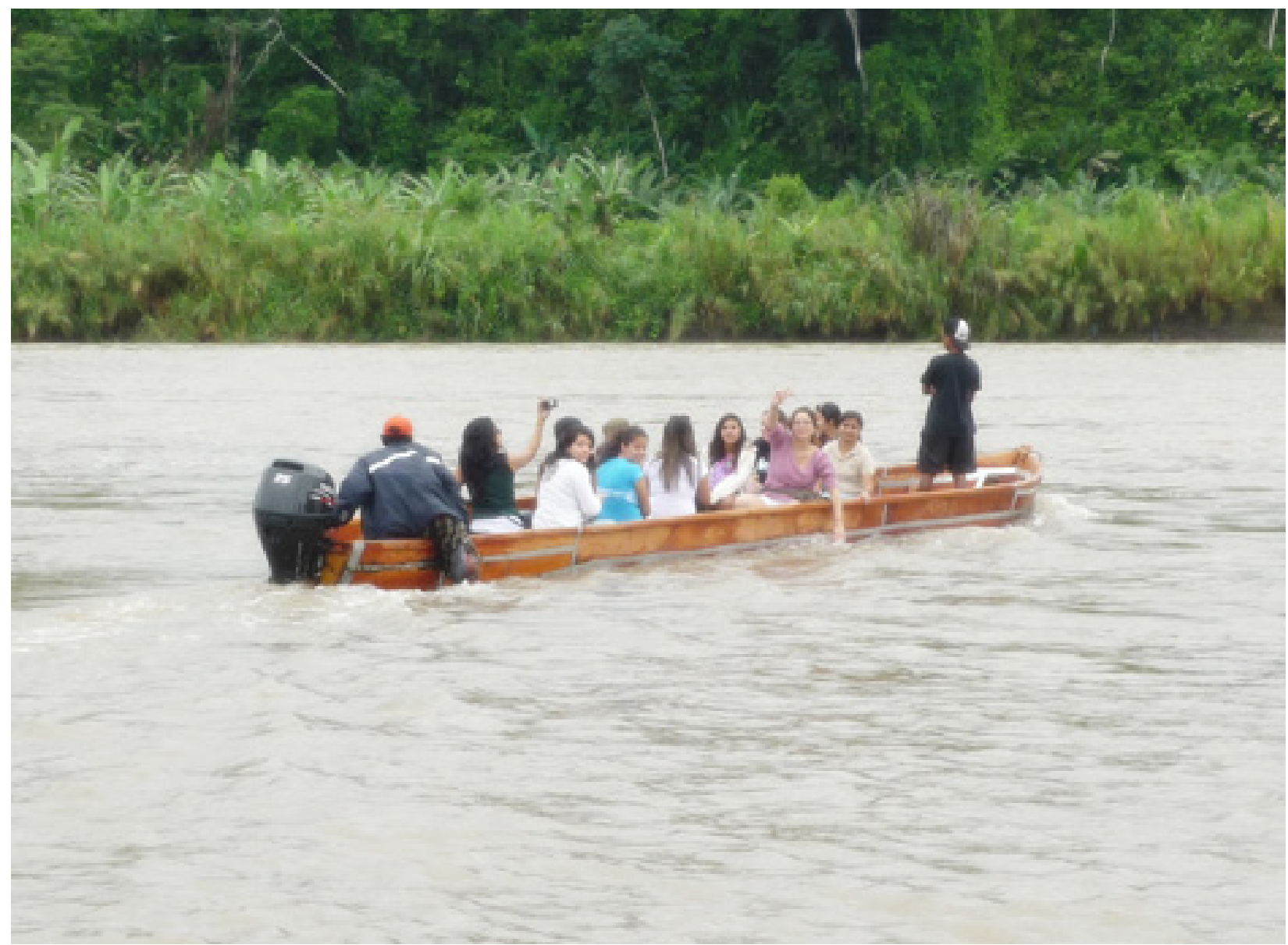

Figura 3. Estudiantes cruzando el río Telire, Talamanca, 2011.

Desde el punto de vista de los valores que motivan el aprendizaje y que son básicos para procurar una educación de calidad (como lo son las competencias para vivir en democracia y para ejercer una ciudadanía activa), se hace necesario determinar cuáles son las necesidades de los y las [sic] estudiantes y de los y las maestras (sujetos todos de aprendizaje) y de qué forma se deben realizar los ajustes requeridos para solventar las deficiencias en los y las [sic] escolares. (Muñoz, 2005, p. 28) 
Está claro, entonces, que para lograr desarrollar las competencias para vivir, se debe replantear la forma en que la enseñanza y la transmisión de conocimientos se ha venido asumiendo: ya no basta con la teoría, si esta no se adapta a la realidad del estudiantado; y tampoco basta si el estudiante y la estudiante no pueden ser capaces de confrontar lo aprendido con la realidad y adaptarlo a las especificidades del entorno. La formación docente es, pues, un reto que demanda procesos que lleven al estudiantado a lograr mirar, reflexionar y ser; en cuanto a lo que se les propone, se necesita más que memorizar teorías o repetir ideas ajenas, que construyan, innoven, transformen y sean. En relación con la formación docente, Flores (1994), citado por Cárdenas (2011), resalta lo siguiente:

El concepto de formación, desarrollado inicialmente en la ilustración, no es hoy día operacionalizable ni sustituible por habilidades y destrezas particulares ni por objetivos específicos de instrucción. Más bien los conocimientos, aprendizajes y habilidades son apenas medios para formarse como ser espiritual. La formación es lo que queda, es el fin perdurable; a diferencia de los demás seres de la naturaleza, "el hombre no es lo que debe ser", como decía Hegel, y por eso la condición de la existencia humana temporal es formarse, integrarse, convertirse en un ser espiritual capaz de romper con lo inmediato y lo particular, y ascender a la universalidad a través del trabajo y de la reflexión filosófica, partiendo de las propias raíces. (p. 2)

Asimismo, Lépiz y Dengo (1999, p. 37), en relación con la filosofía de la educación de Vicenzi (1940), enfatizan en que se debe "darle al maestro una 'concepción total de la vida', sin la cual no está en condiciones para 'transmitir cultura y acrecentarla y dirigirla"; en ese sentido , por lo tanto, brindarle al estudiantado experiencias educativas fuera del aula es imperante en una búsqueda del mejoramiento, ya que estas son necesarias para que pueda lograr una concepción total de la vida a través de su formación educativa y que no la perciba solamente como un hecho que ocurre artificialmente en los libros de textos y los relatos docentes.

Cárdenas (2011) refuerza la postura planteada en los párrafos anteriores sobre la formación de formadores y formadoras, y menciona diferentes aspectos que no pueden desligarse de esta:

Formar personas requiere compromisos éticos, políticos, sociales, culturales y esto es posibles [sic] desde el amor el cual crea seguridad y abre futuro. Cuando un docente vive su profesión no como un saber que le crea un poder- dictador de clase- o como una obligación que tiene que cumplir -trabajo-, sino como un proyecto de vida donde todos los días se aprende, se mejora y se descubre ayudando a reconocer las capacidades, habilidades y destrezas para la vida, está dando sentido a su misión, está educando, está ayudando a "ser", está humanizando. Y este debe ser el sentido de la formación docente. (p. 5) 
Es así como la autora agrega matices fundamentales en la formación de personas formadoras, mencionando términos tales como el amor, vivencia, aprendizaje continuo y humanización. Y en este punto, es importante recalcar que no se puede desarrollar en el estudiantado lo anterior, si no se modela y se da el ejemplo cuando se asume el acto educativo. En la misma línea, L. E. Flores, G. A. Flores, Jiménez, Madrigal y Perearnau (2009) plantean que:

Asumir el nuevo paradigma significa situarse en una pedagogía alternativa, en una biopedagogía del aprendizaje con visión cósmica que nos relaciona con la totalidad. En el paradigma emergente se concibe la educación como opción emancipadora y hay una ruptura con el concepto mecanicista del aprendizaje. (p. 19)

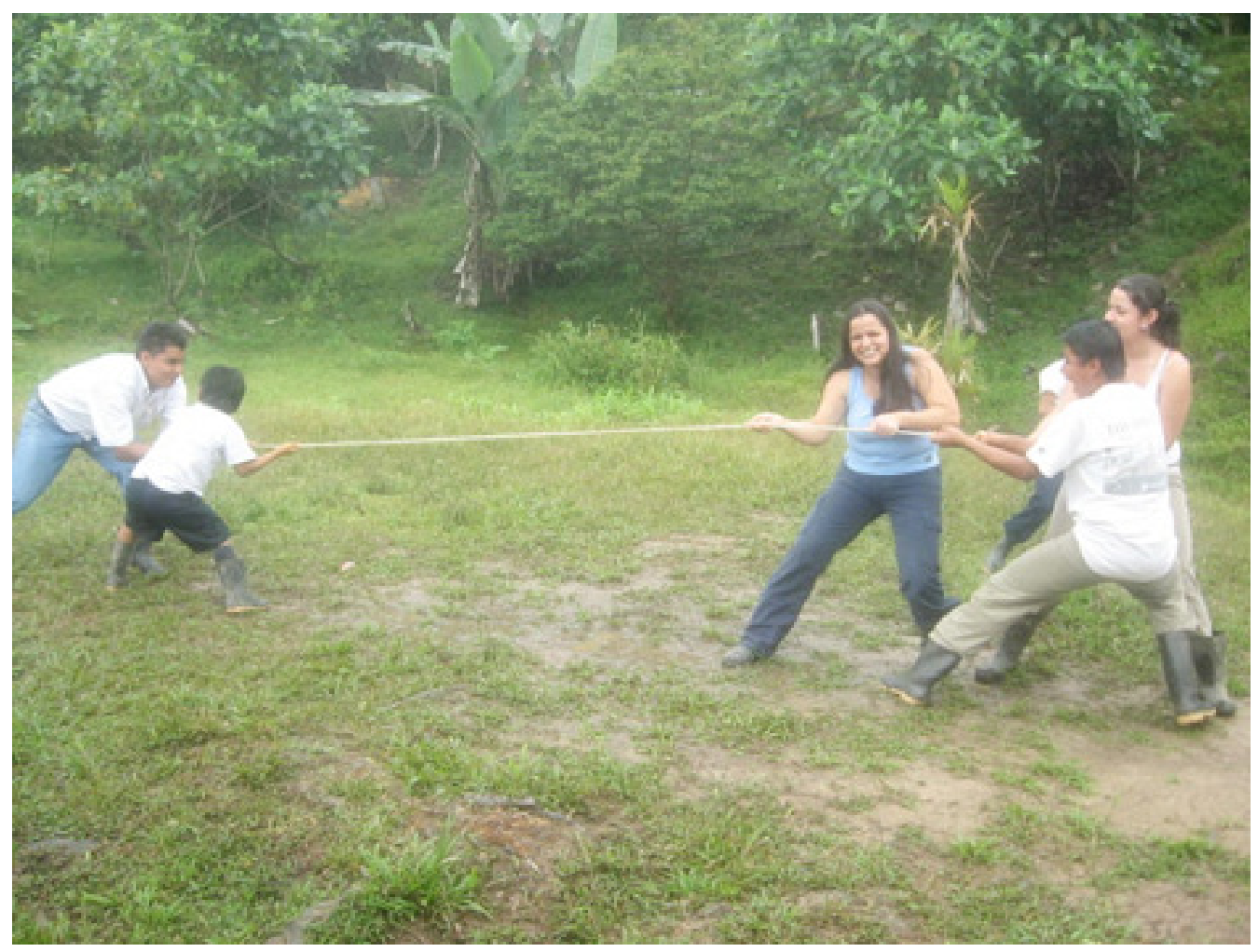

Figura 4. Talleres recreativos con estudiantes de la comunidad de Alto Urén, Talamanca, 2007.

Por lo anterior, al valorar opciones para una nueva propuesta educativa que logre ampliar las estrategias utilizadas en la educación superior, se deben buscar actividades que propicien en el alumnado un encuentro consigo mismo, donde tenga la opción de ser partícipe de su proceso de enseñanza y aprendizaje, y ser parte de la aventura de aprender y ejercer así su derecho a "ser" (ver figura 4). En esta línea, Muñoz (2005) menciona: 
Una educación neutra o al servicio de otras necesidades, [sic] no puede desarrollar la personalidad respetuosa a los derechos humanos, porque esa neutralidad es potencial y actualmente convalidadora de desigualdades. Por eso sostenemos, con Freire, que la educación constituye un espacio de libertad para el ejercicio y el aprendizaje de todos los derechos, responsabilidades y capacidades humanas. (p. 16)

El espacio de libertad del cual se habla, tal y como se había mencionado anteriormente, no se puede lograr sin docentes que propicien estrategias de enseñanza y aprendizaje tendientes a despertar en el estudiantado el gusto por la docencia, la pasión por la profesión y, sobre todo, el deseo de descubrir su entorno y así resignificarse como personas únicas y valiosas. Al respecto, Vicenzi (1940), citado por Lépiz y Dengo (1999), afirma que:

El docente "no enseña como quien entierra bloques de piedra. Anima. Si no es animador, no es un maestro: es una máquina sin vida"y agrega que sin proceso, sin fuerza, sin acción, no hay pedagogía posible, ya que el conocimiento no podría existir sin ritmo. (p. 49)

Por lo tanto, se puede rescatar la importancia de plantear nuevas formas de enseñanza que le den, a la labor de aula, la fuerza y acción necesaria para animar a cada uno y cada una de los estudiantes y las estudiantes que han escogido ser formadores y formadoras como opción de vida. Es momento de reflexionar sobre los resultados obtenidos, haciendo lo que siempre se ha hecho y empezar a soñar con los posibles resultados, si nos atrevemos a ir por nuevos caminos y trazar otros rumbos cuando acompañamos al estudiantado en su proceso de enseñanza y aprendizaje.

\section{Contextualización teórica}

Luego de un repaso de diferentes posturas sobre características deseables a lograr en la formación de formadores y la búsqueda de respuestas para la urgente necesidad de un cambio en la forma en que se desarrollan las clases en las aulas, se propone la expedición como herramienta pedagógica, ya que brinda las condiciones para lograr formar futuros docentes con características como las mencionadas en el apartado anterior (ver figura 5). Al respecto, Laverde (s. f.), citada por Suárez (2003), menciona lo siguiente:

Y me pregunté aquel día qué era la escuela. Acaso, ¿es el cuarto frío y oscuro del que me han hablado algunas veces? $\mathrm{O}$ tal vez ... ¿ ies eso que algunos describen como una puerta sin salida, como una constante máquina de repetición? ¿Qué es entonces? No sé, ya me he aburrido un poco con tantas visiones, ya me han cansado las palabras de aquellos que no quieren que yo la descubra. Por eso quiero encontrar su verdadero significado, su verdadero sentido, ese sentido perdido, ese sentido de escuela, esa realidad de las que no nos enteramos. Y sin más espera, he salido a recorrer los pasos que no percibo, los pasos de aquellos que se encuentran en ese desconocido espacio. (p. 185) 
URL: http://www.una.ac.cr/educare

CORREO: educare@una.cr

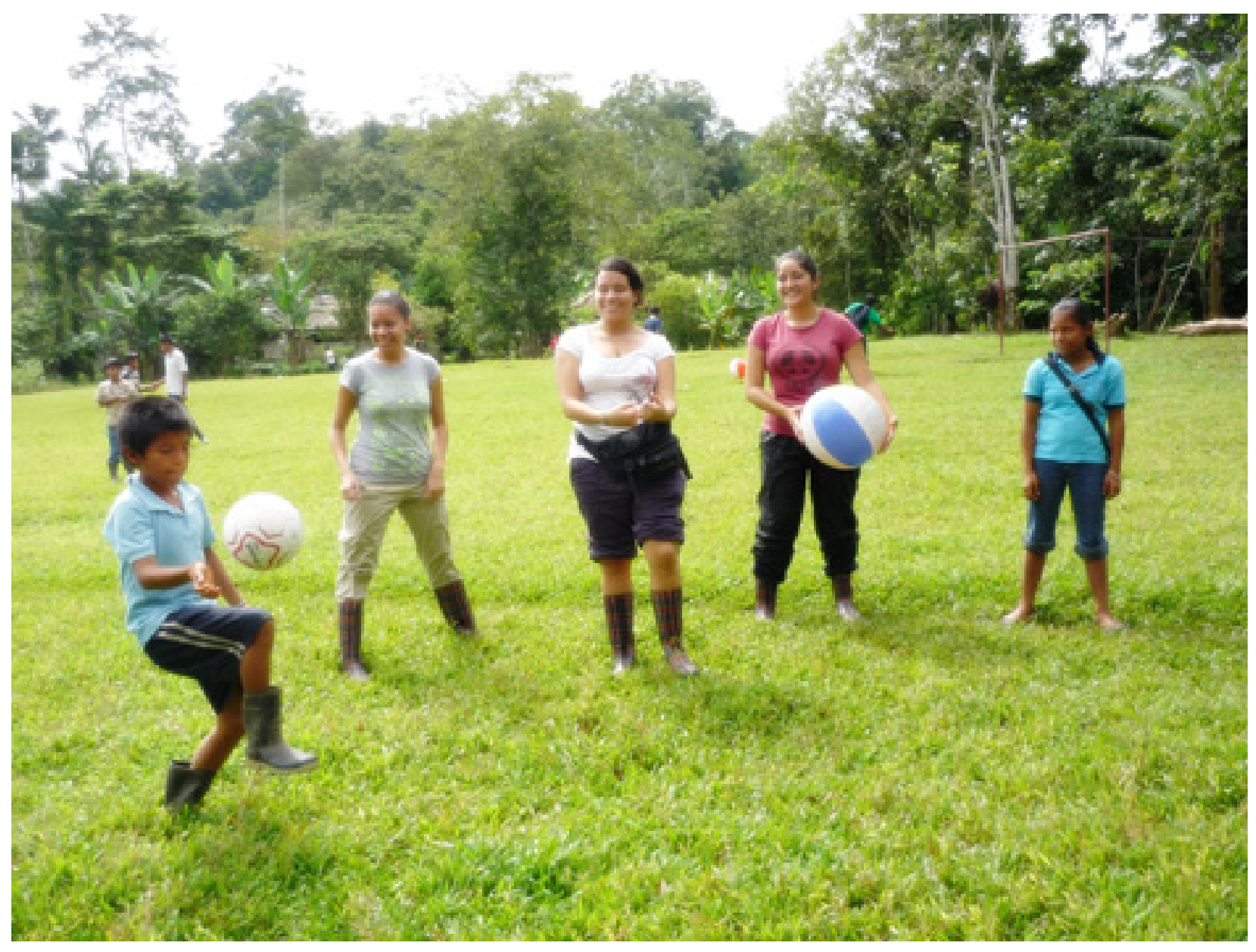

Figura 5. Talleres recreativos con estudiantes de la comunidad de Kachabri, Talamanca, 2011.

En las palabras citadas, se retrata la realidad y se propone un cambio: el viajar, el conocer otras realidades educativas, otros contextos y, así, descubrir, de primera mano, los secretos que han tratado de ser contados durante las sesiones de clase; pero los cuales, por su condición teórica, no han podido ser vivenciados. El viaje es la esencia de la propuesta. A esto, el pensamiento de Unda (2008, p. 1) aporta elementos importantes, al mencionar que viajar va más allá de los conceptos de recreación, ocio, turismo o trabajo, "en la Expedición Pedagógica son diferentes, se conectan con el pensamiento, con el asombro, con la posibilidad de experimentar otras miradas sobre nosotros mismos, de ver, de nombrar aquello que no había sido visto ni nombrado", es decir, se convierte en el acto que le da vida a lo que muchas veces no tenía significado y le brinda al estudiantado opciones de crecimiento en muchas áreas de su persona, que posteriormente le serán de utilidad al ejercer la profesión docente. 


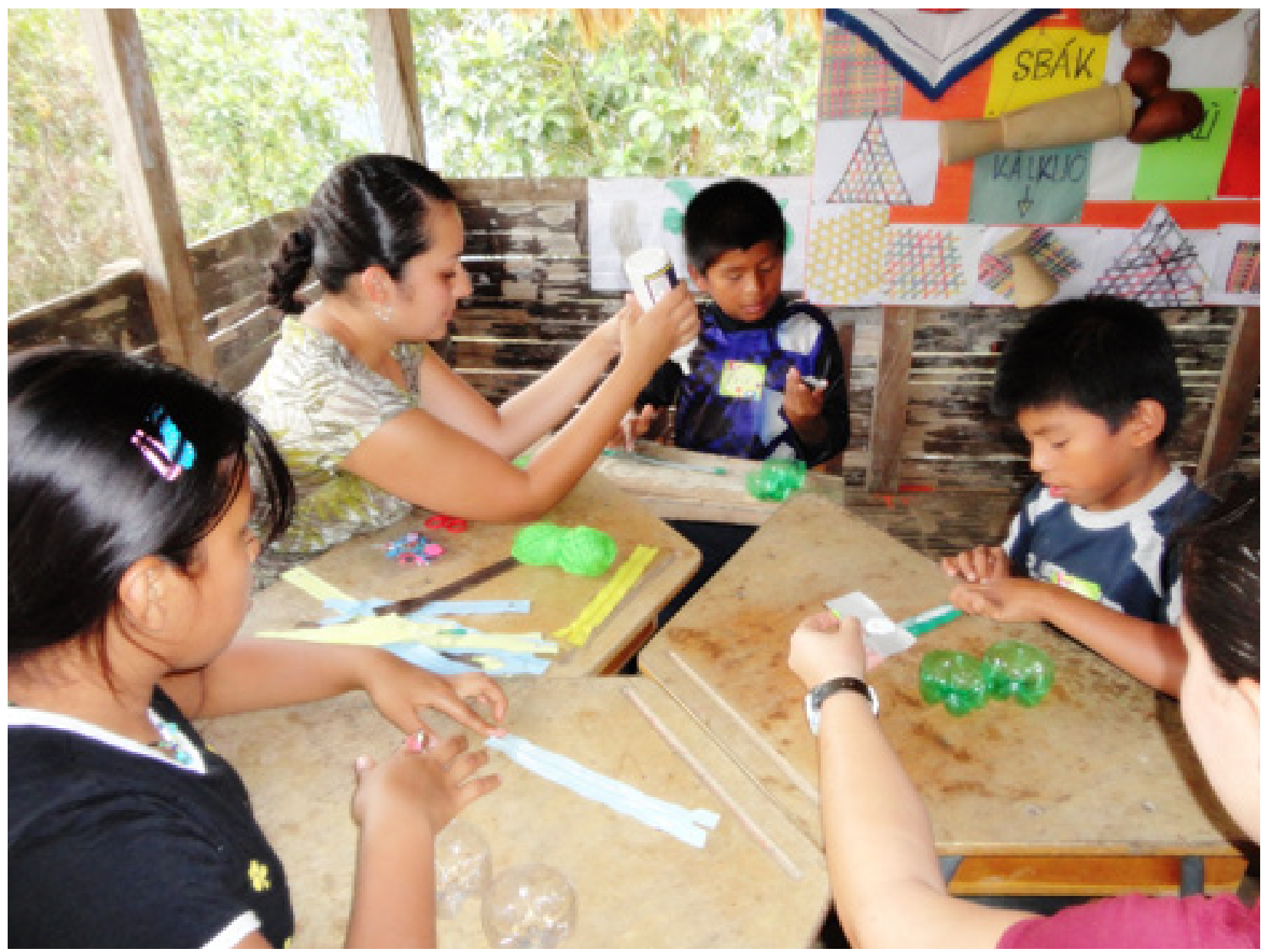

Figura 6. Talleres con estudiantes de la comunidad de Alto Urén, Talamanca, 2007.

En la misma línea, Rodríguez y Forero (2011), comparten que "viajar implica anticiparse, moverse, salir del sitio, emprender una acción; viajar reclama goce, disfrute, placer" y agregan lo siguiente:

Solo viajando se conoce y se reconoce-[sic] los territorios, los sujetos, los saberes, las realidades. Se habla de viajar como un desplazamiento que es físico y de pensamiento. Se viaja físicamente para tener una percepción cercana con todos nuestros sentidos de los acontecimientos pedagógicos y viajamos con y por [sic] el pensamiento para flexibilizarlo y dar lugar a comprender cuantas diferencias, riquezas, dolor, esfuerzo, se encierran en nuestra geografía y en nuestras escuelas. Las prácticas se deben incorporar a un acto de pensamiento, ya que esta se valora como experiencia cuando es reflexionada. (p. 2) 
URL: http://www.una.ac.cr/educare

CORREO: educare@una.cr

Lo anterior refuerza el planteamiento de que la expedición pedagógica brinda muchas posibilidades para lograr un cambio cualitativo en los procesos de formación en la educación superior, abriendo un abanico de opciones para que el estudiantado conozca el entorno, se humanice, crezca en libertad y logre llevar a cabo procesos autónomos de pensamiento $y$, sobre todo, brinda un espacio para reinventarse $y$, de esta manera, lograr resignificarse como personas y futuros docentes (ver figura 6). Al respecto, Hernández (2009) indica que:

Con las expediciones se realiza un viaje en el cual se descubre, se recorre y se transita por la cotidianidad del maestro y de la escuela convirtiéndose en un escenario para hablar de realidades re-significando el que-hacer docente y a su vez la producción de conocimiento, a través de las prácticas educativas exitosas que muestran la diversidad sobre los recursos utilizados para ese hacer por los docentes en el desarrollo de sus actividades individuales y colectivas que tienen dentro y fuera de sus aulas en los centros escolares así como en otros contextos. (p. 7)

Este viaje debe llevar a despertar en el estudiantado el asombro, la pasión y guiarlo a asumir la responsabilidad de ser y existir y todo lo que esto conlleva; es imperante que se conozca el entorno, se estudie, se observe, se mire, se viva y se palpe. No debe bastar con escuchar sobre otras realidades, es responsabilidad de los formadores de formadores de exponer al estudiantado a estas realidades y proveer oportunidades de conocer más allá de si mismo. El futuro docente debe ser capaz de comprender que el acto educativo está compuesto por innumerables variables, pero que el contexto es una de las prioridades por atender, para poder lograr con éxito los objetivos propuestos en el salón de clases. Sobre lo anterior, Adalgiza et al. (2005) afirman que:

Viajar significa abrirse a nuevos mundos, es llegar y encontrar cosas diferentes; es desplazarse, es movimiento de sentimientos, de acción y de pensamiento; es tomar decisiones en cuanto a dónde, cómo, cuándo y con qué y con quién hacer el viaje; es encuentro con quienes viajas y con quienes vas a encontrar; es enriquecimiento porque aprendes del conocimiento de otros y otras; es sorprenderse ante la expectativa creada y lo encontrado; es aventura porque propicia nuevos encuentros; es reconocerse y diferenciarse de otros y con otros, en fin viajar invita a SER. (p. 4)

Lo anterior se refuerza con las palabras de Rodríguez y Forero (2011), quienes contribuyen a comprender un poco más el significado real de viajar y lo que puede aportar a la labor docente: 
La idea de viaje invita a mirar a ser mirado a través de las experiencias del otro y de lo otro, es reconocer, recorrer, escudriñar, sorprenderse frente a lo que aparentemente se muestra como cotidiano, pero que en los ojos y en el sentir de los maestros expedicionarios adquiere otros sentidos. (p. 3)

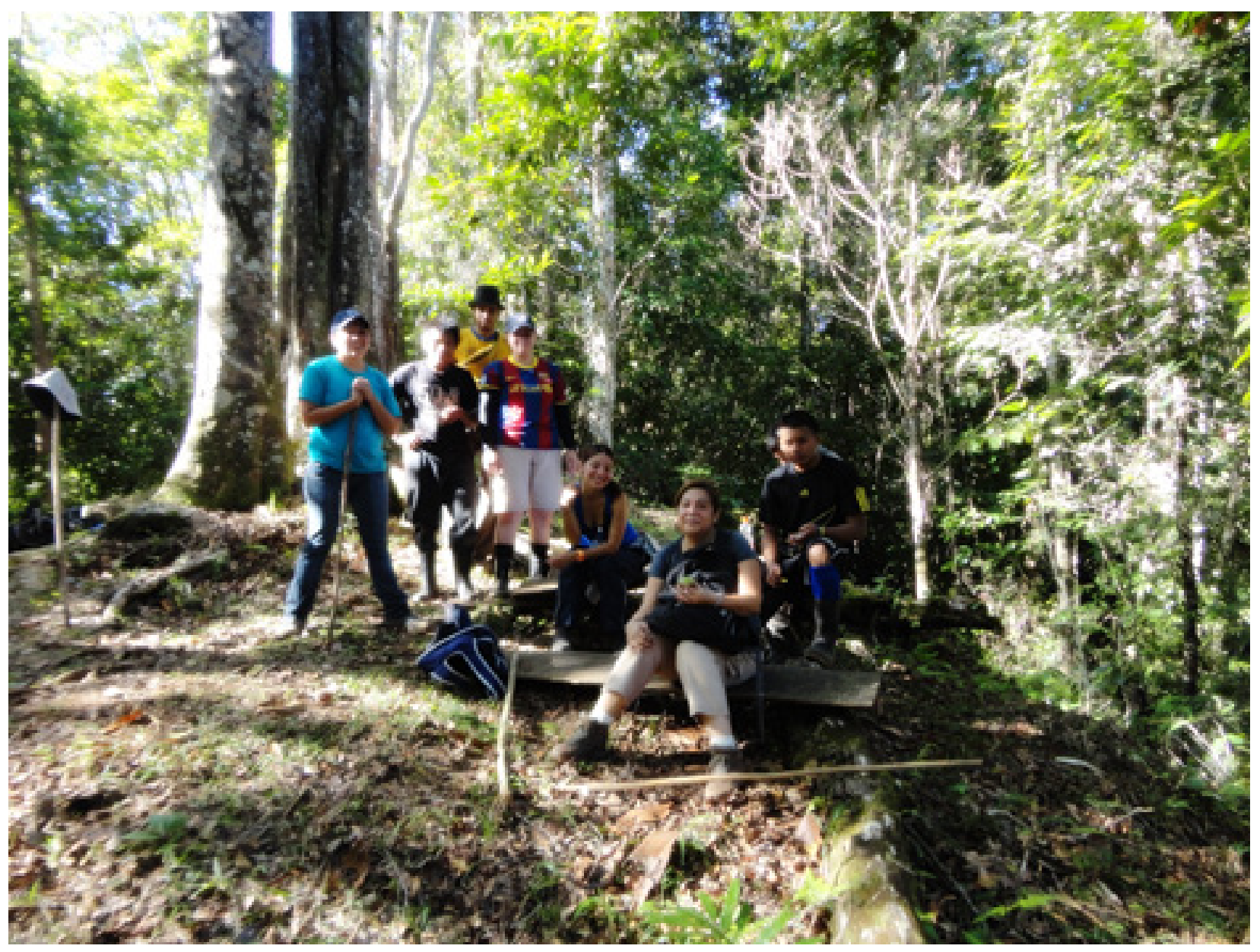

Figura 7. Alto Urén, Talamanca, 2011.

Es importante hacer notar que la colectividad aporta muchas ventajas al viaje individual que se hace durante el proceso de formación, y mucho más porque al presentarle al grupo de aprendientes una experiencia de aprendizaje diferente, la cual se desarrolla en un contexto ajeno y que permite que surja la incertidumbre, puede lograrse la trasformación personal a través del trabajo grupal (ver figura 7). Al respecto, Suárez (2003) comenta: 
Estamos aquí, a las puertas del espacio, para el encuentro, para el intercambio de experiencias, para un diálogo de saberes; se trata de aprender haciendo, de hacer preguntando, de preguntar sonriendo, de sonreír entendiendo, de entender escuchando, de escuchar haciendo. (p. 91)

La expedición nos brinda tantas oportunidades de crecimiento, que luego de experimentarla, se vuelve imprescindible como herramienta pedagógica en el aula. Resulta impresionante la transformación que puede provocar en la mayoría del estudiantado, y observar los logros que, en ocasiones, no se pueden alcanzar luego de todo un semestre de clases. Es una oportunidad de transformación personal para el docente y la docente, quien también se reconstruye durante el viaje y se reinventa junto con el estudiantado. Asímismo, Luna (2011) comparte que la expedición:

Es poner nuestros saberes en un proceso de construcción colectiva permanente y crítica sobre lo que cada uno produce, enriqueciéndolo y transformándolo pero dejando que cada uno conserve los sonidos propios de su voz, sus colores, matices y riquezas propias. Porque no se trata de homogenizar ni de unificar unas prácticas; por el contrario, es dar paso a la pluralidad de ideas a nuevas, otras formas de construir tanto en lo individual como en lo colectivo. (p. 3)

Es un proceso en el que cada persona aporta y el colectivo se enriquece de la experiencia, se conocen las fortalezas y debilidades, se reconocen habilidades que no se conocían en los participantes y las participantes, y se comparte la esencia misma de la persona en relación con otros. En esa misma línea, es importante también resaltar lo que Rodríguez y Forero (2011) mencionan:

El viajero en expedición no es un simple explorador, es un maestro investigador que constantemente está en viaje por el pensamiento, el conocimiento y la escritura. Es un viajero que explora el territorio hacia adentro es decir continuamente está reflexionando sobre su quehacer Pedagógico y mediante la interacción con otros maestros viajeros, reconoce otras prácticas y visibilizar otros modos de ser maestro. (p. 5)

Las experiencias que se vivirán nunca serán iguales, ya que cada estudiante las abordará de acuerdo con su forma de ver e interpretar el mundo y las procesará de forma diferente que los otros (ver figura 8). En cuanto a ello, Rodríguez y Forero (2011) comparten que:

Cada viajero traza su ruta, aunque se vaya por el mismo sendero que otros, no hace el mismo recorrido, aunque el camino sea compartido, cada expedicionario determina su trayecto con lo que tiene en sí, mira las cosas según sus posibilidades, mirada que conjuga intereses, percepciones, y emociones. (p. 5) 


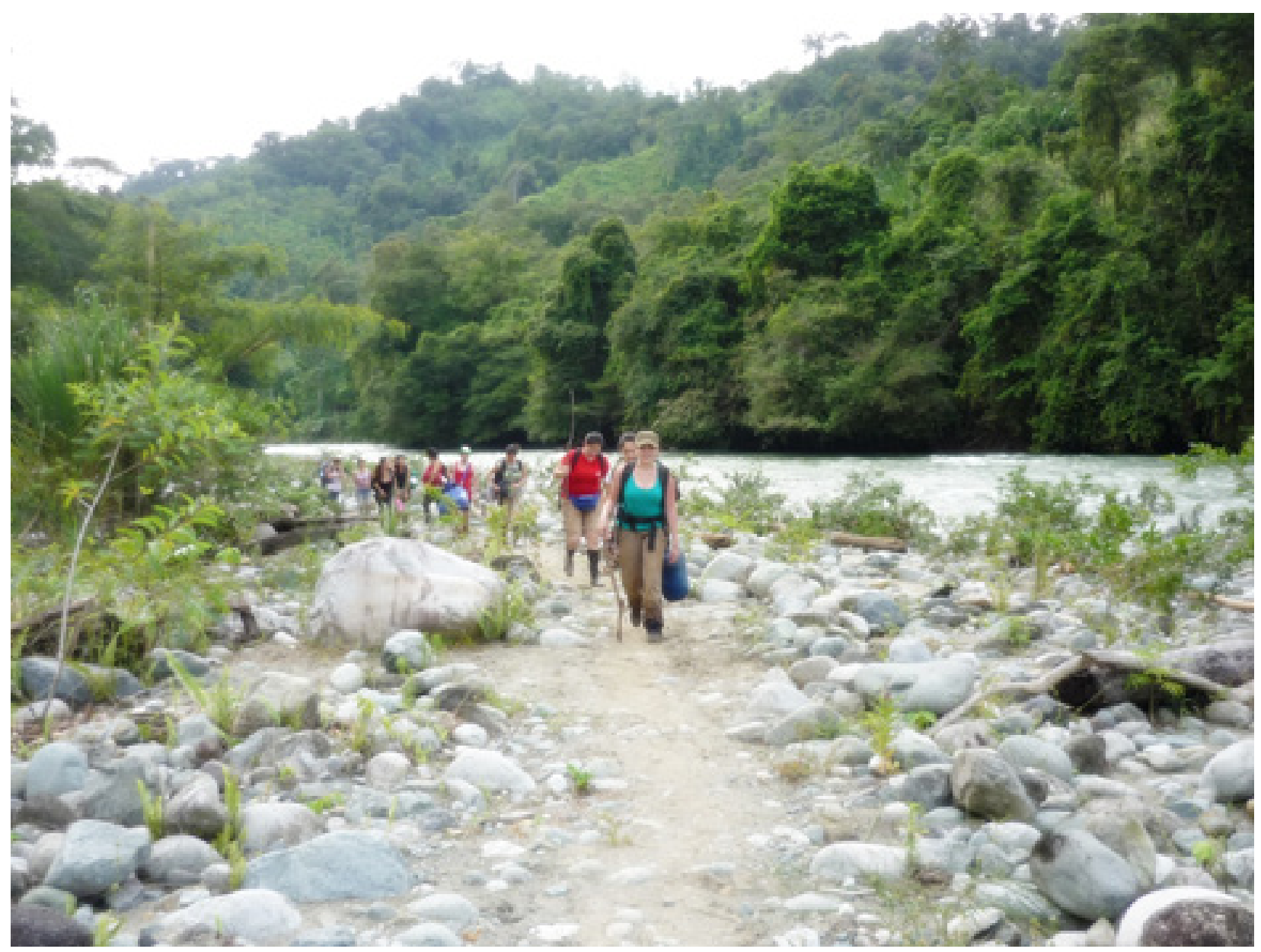

Figura 8. Kachabri, Talamanca, 2011.

Y aunque al final todos llegan al mismo lugar, el recorrido y transformación del individuo es único, cada persona descubrirá lo que estaba destinado a descubrir, cada una tendrá una historia única que contar y se transformará en aquellas áreas donde era necesario que lo hiciera. El colectivo se conformará también un una entidad con características propias, que luego aportará a las partes un cúmulo de experiencias inolvidables, ya que no podemos ser sino lo que somos en relación con los demás y, durante las expediciones pedagógicas, es primordial el vínculo que se crea en el grupo y la dinámica que se forja conforme la experiencia se desarrolla.

\section{Estrategia metodológica: la expedición pedagógica}

La expedición pedagógica surge como una inquietud durante mis primeros pasos como docente universitaria, luego de conocer pioneras en esta labor, quienes, mediante el modelaje y la generosidad de compartir los secretos que guarda el viaje como experiencia de formación en la educación superior, me encantaron con la idea y me dieron el impulso para empezar 
a practicarla de una manera rústica al inicio, para luego poder aplicarla de una forma más organizada, metódica y con objetivos y metas claras.

Es importante recalcar que, al igual que cualquier intento de innovar y de asumir que es necesario ampliar el horizonte que tenemos cuando se tiene la responsabilidad de educar, la expedición es una actividad que demanda mucha organización, requiere que cada etapa sea asumida de forma programada y que el estudiantado participe en los eventos previos y posteriores a esta.

No se puede asumir como una experiencia que no tiene antes ni después, ni tampoco dejar de involucrar a la totalidad de participantes, ya que de esto depende gran parte de que logremos el crecimiento y transformación colectiva e individual. En relación con esto, Unda (2008) comparte lo siguiente:

La preparación de los viajes no es una tarea menor. Pasa por identificar condiciones de lugar, de tiempo, recorridos, itinerarios, actividades a realizar y funcionamiento logístico, pero se dedica especialmente a la formación de los expedicionarios: viajeros, anfitriones y organizadores. Es una preparación para el encuentro, para el intercambio, para la observación, para sentir, escuchar y amplificar las voces de quienes no han sido oídos, las voces de maestros y maestras, de niños y niñas, jóvenes, hombres y mujeres que, desde sus territorios, hablan de sus mundos, los problematizan y adelantan propuestas inusitadas de actuación. (p. 1)

En esta línea, cada una de las experiencias vividas a partir del 2005, como parte de la labor docente y de extensión realizada como académica de la DEB, y cuya sistematización lleva a la construcción del presente artículo, se desarrollaron con el objetivo de brindar al estudiantado y cuerpo docente, experiencias de aprendizaje donde la realidad asume un papel preponderante en la formación académica. Esto, debido a que el participante y la participante deben interrelacionarse reflexivamente en contextos ajenos a su convivencia cotidiana, con el fin de que logren enriquecerse con los conocimientos que les aporta esta vivencia y los que construya a partir de su análisis reflexivo, buscando, durante cada una de las etapas, que las siguientes metas fueran alcanzadas:

1. Ofrecer espacios de formación y desarrollo profesional a partir de la vivencia y convivencia entre miembros de comunidades educativas de diferentes lugares.

2. Propiciar encuentros académico-formativos entre actores sociales vinculados a la educación e inspirados en experiencias educativas innovadoras donde se favorece la formación para la vida

3. Favorecer el intercambio académico entre futuros educadores, de manera que se aprovechen los escenarios naturales del ejercicio profesional, para generar aprendizajes significativos e innovación en los centros escolares y comunidades. 
4. Promover transformaciones en el ejercicio profesional a partir del intercambio.

5. Implementar ideas a partir de las experiencias vividas.

Las expediciones pedagógicas se realizaron a diferentes comunidades ubicadas en zonas rurales costarricenses. Los participantes y las participantes de las diferentes experiencias desarrolladas fueron en total ciento sesenta y cinco estudiantes y docentes de las diferentes carreras que oferta la DEB.

A inicios del 2013, se aplicó un cuestionario en línea a las personas participantes, con la finalidad de valorar la expedición pedagógica a partir de su propia vivencia. Es importante enfatizar que solamente se obtuvo respuesta de treinta y siete participantes, por lo tanto, los hallazgos están basados en los participantes reales.

\section{Resultados más relevantes derivados de esta experiencia}

En relación con los aspectos que más impactaron positivamente su forma de percibir la realidad, el $88 \%$ de los estudiantes y las estudiantes mencionan que el enfrentarse a situaciones diferentes a las de su vida diaria; el $76 \%$ del estudiantado agrega que las condiciones de las comunidades que se visitaron; seguidos por un $72 \%$ que señala como mayor impacto, la interacción con los niños y las niñas y, por último, un 68\% indica la interacción con la comunidad.

De los datos derivados de los participantes de la experiencia, más de un $81 \%$ mencionan que después de la experiencia vivida, consideran que las expediciones son muy importantes en relación con los siguientes aspectos, tal y como se observa en la figura 9:

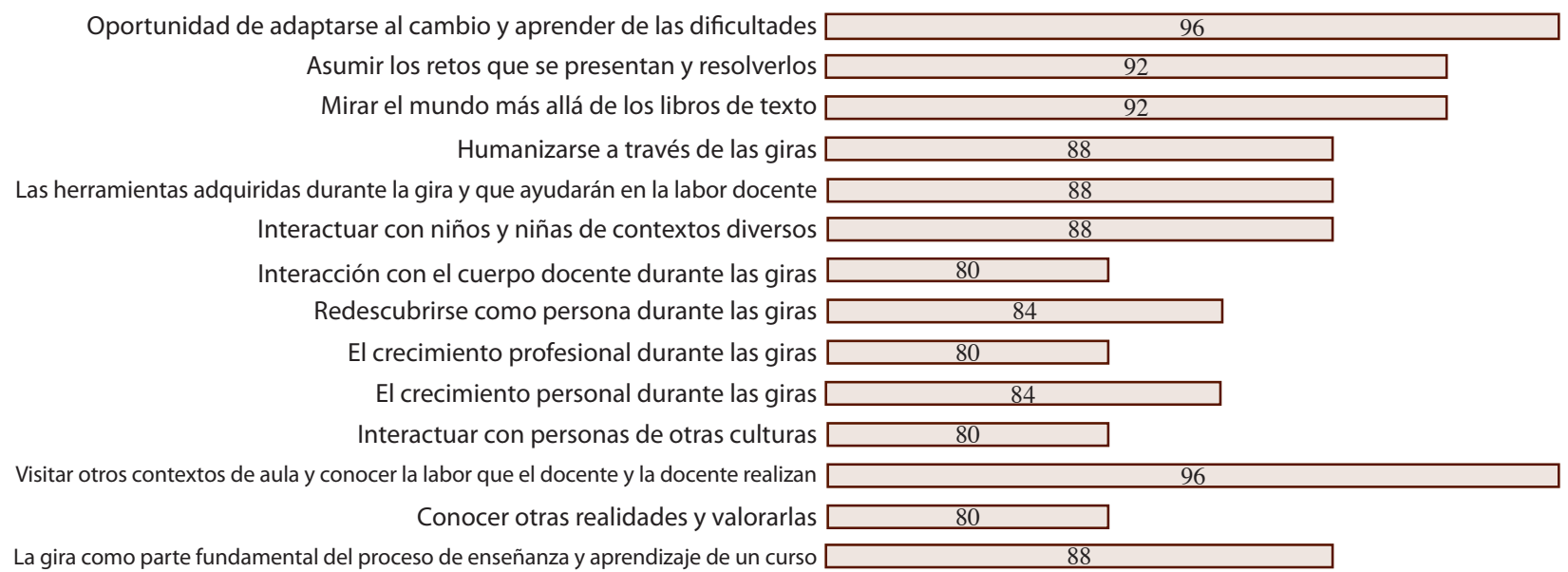

Figura 9. Distribución procentual de aspectos valorados como importantes por las personas participantes en las expediciones. 
URL: http://www.una.ac.cr/educare

CORREO: educare@una.cr

Lo anterior refuerza lo planteado al inicio, en cuanto a que la expedición pedagógica brinda herramientas al estudiantado, fortalece su proceso de enseñanza y aprendizaje, y ensancha sus horizontes como personas y como futuros docentes y futuras docentes. Enfatizan en aspectos tales como la oportunidad de desarrollar herramientas para adaptarse al cambio, visitar otros contextos de aula, asumir retos que se presentan y resolverlos entre otros. Muchas veces la teoría por sí sola no podría llevar a cabo estos procesos en el estudiantado, o requeriría muchísimo más tiempo del que disponemos para lograr que desarrollen ciertas habilidades deseables solamente con clases que se imparten en el aula universitaria; por lo tanto, el brindarles oportunidades de crecimiento como las que la expedición provee es una responsabilidad de los formadores y las formadoras. El lograr que los aspectos mencionados puedan alcanzarse no es tarea fácil, pues requiere de un esfuerzo extra por parte de los docentes y las docentes; pero ese esfuerzo no solamente trae ganancias en cuanto al logro de objetivos propuestos en un curso, sino que también contribuye a mantenernos "vivos" como educadores y educadores, aporta energía vital a nuestra labor docente y nos transforma como profesionales y como seres humanos.

Los participantes y las participantes de la experiencia, detallan lo anterior mediante las siguientes afirmaciones acerca de la experiencia:

"El conocer diversas realidades de las cuales no se está acostumbrado interactuar, el ir más allá de la teoría de las cuatro paredes que no permiten conocer la realidad de muchos pueblos, crecer como persona y profesional valorando lo que se tiene y tener el gusto de llevar a cabo la labor docente de la mejor forma posible teniendo presente aprendemos junto a nuestros estudiantes, sus familias y contexto, aspecto que se aprende en gran medida en estas giras pues ampliamos la visión de queso estudiantes no son en singular sino en un sentido plural pues es un ser integral que cuenta con una realidad e historia de vida las cuales estarán presentes durante todo su vida y por ende en sus procesos de aprendizajes".

En relación con otros aspectos mencionados en las respuestas del instrumento, relacionados con la organización y el trabajo en grupo, se observa que lo que más se valora de la experiencia vivida, por parte del estudiantado, es:

"El crecimiento como persona, la sensibilidad que se desarrolla al compartir con personas que llevan un estilo de vida muy diferente al mío, los lazos de confianza que se establecieron con las y los [sic] compañeros de curso y la habilidad de organizarse que se requiere previa a la gira, tomando en cuenta que todos(as) tenemos gustos y hábitos distintos".

Además, en otras respuestas, se retoman aspectos de crecimiento profesional, enriquecimiento de las relaciones interpersonales, la adquisición de herramientas diferentes para hacerle frente a distintos problemas o situaciones y el conocimiento de diferentes contextos educativos. 
En cuanto a la meta de ir más allá del aula universitaria como espacio físico para realizar el acto de enseñanza y aprendizaje, responden que:

"Aprende uno muchas cosas como persona y docente, se obtienen muchos conocimientos, es una experiencia realmente increíble, hace falta hacer algo diferente a leer un libro, se aprende mejor de manera constructiva".

La totalidad de estudiantes que se encuentra laborando, (35\%) manifiesta que la experiencia vivida durante la expedición pedagógica les ha ayudado en su labor docente. De esta forma, agregan que los aspectos que más les han aportado de las expediciones a su labor son las planteadas en la tabla 1.

Tabla 1

Aspectos en los cuales la expedición pedagógica le ha aportado a la labor docente de quienes participaron en las giras

- Tener presente la importancia de llevar a cabo un trabajo en equipo

- No limitarse con los pequeños cambios que se pueden llevar a cabo dentro del aula para generar un aprendizaje significativo

- Reconocer un espacio para que el docente expanda sus conocimientos y que aprenda de esas experiencias para ponerlas en práctica con otros estudias y compartirlas con colegas

- Entender que se aprende mucho más en un contexto real que solo por un par de libros

- Adaptarse a situaciones y buscar soluciones reales

- Saber de la variedad de herramientas que se pueden utilizar para propiciar un aprendizaje más significativo

- Lograr una mejor comprensión de las diferencias de contexto e incentivo a la convivencia intercultural entre la comunidad educativa

- Permitir ser un docente observador y más empático y humano ante las necesidades de los niños y las niñas

- Respetar las diferentes culturas y formas de vida

- Valorar más el ambiente y los materiales que se tienen en la escuela donde uno está laborando

- Valorar lo que se tiene

En los aspectos mencionados por las personas participantes de la experiencia, se puede visualizar cómo, desde su perspectiva, la expedición pedagógica es un recurso viable y valorado, que aporta no solamente a su crecimiento profesional, sino también personal, y les brinda un aprendizaje innovador y significativo que impactará a futuro el rol docente que desempeñen. 
URL: http://www.una.ac.cr/educare

CORREO: educare@una.cr

\section{Reflexiones finales}

Los cambios en los paradigmas educativos muchas veces son difíciles, conllevan un esfuerzo extra en nuestras labores cotidianas, tal y como lo comentan Torío et al. (2010):

En suma, el reto del cambio metodológico nos obliga a revisar concienzudamente muchas de las dinámicas y formas de organización y de trabajo de la institución universitaria, a la vez que nos ofrece interesantes posibilidades desde el punto de vista pedagógico y didáctico. (p. 251)

La expedición pedagógica se ha convertido en una experiencia única desde la perspectiva estudiantil, pues después de cada experiencia vivida señalan que les aporta nuevos conocimientos que inciden en su crecimiento personal y profesional. Se puede decir que el objetivo y metas propuestas se lograron, y se pudieron establecer las ventajas que brinda la herramienta como parte del proceso de enseñanza y aprendizaje en el ámbito universitario.

Los resultados obtenidos en el presente estudio sugieren que la expedición pedagógica podría representar un impacto significativo en la forma de conceptualizar la labor docente por parte del estudiantado, por lo que se debe enfocar la mirada en las posibilidades que ofrece y avanzar, tal y como lo mencionan Flores et al. (2009), quienes señalan que una manera de estimular la existencia y poder trascender es mediante la resignificación de la vida y la historia, lo cual es una responsabilidad intrínseca a la labor de la docencia, donde se debe mirar siempre hacía adelante y resignificar la vida junto con el estudiantado.

En esta línea, el estudiantado menciona que lo vivido en este tipo de experiencias le ayudó a ver la vida de forma diferente, a poner en práctica todo lo que aprende y a hacerlo cada vez mejor. Enfatizan, además, que a nivel de grupo aprenden a convivir más y conocerse, aceptándose unos a otros.

Se debe entender que la expedición pedagógica es una herramienta que debe ser utilizada de forma organizada, con objetivos y en conjunto con muchas otras estrategias de enseñanza y aprendizaje. De ser implementada con éxito, le dejará al estudiantado, en palabras de los participantes y las participantes "un sabor de compromiso, motivación y positivismo".

Finalmente, quisiera mencionar las palabras de Withman (2012), quien nos reta a crecer cada día un poco más, a soñar y perseverar; a ser protagonistas de nuestra vida con orgullo y sin miedo, afirmando que no hay que permitir que la vida pase sin ser vivida.

Al respecto, a los lectores y lectoras les insto a ser partícipes, con el cuerpo y con el pensamiento, de la aventura de enseñar y aprender, de explorar nuevos horizontes y conquistar territorios inexplorados. Nuestra responsabilidad como formadores de formadores es inmensa y debemos honrarla cada vez que creamos conocimiento con el estudiantado durante las sesiones de clases. Viajemos, entonces, llevemos la universidad a otros lugares y, con ello, disfrutemos de los tesoros que se esconden más allá de los linderos del campus. 


\section{Referencias}

Adalgiza L., Ramírez, M., Beltrán, P., Barraza, A., Pardo, O. L., Órdoñez, N. y Luna, R. E. (julio, 2005). ¿Para qué se hace una red? Para pescar, para fortalecernos y para producir conocimiento. Trabajo presentado para el IV Encuentro Iberoamericano de Colectivos Escolares y Redes de Profesores que hacen investigación en sus escuelas, Porto Alegre, Brasil. Recuperado de http://ensino.univates.br/ 4iberoamericano/trabalhos/trabalho285.pdf

Cárdenas, S. (julio, 2011). La expedición pedagógica y la formación de maestros. Trabajo presentado en el $\mathrm{VI}$ encuentro iberoamericano de colectivos escolares y redes de maestros/as que hacen investigación e innovación desde la escuela, Córdoba, Argentina. Recuperado de http://www.colectivoeducadores.org.ar/cd 6to encuentro/ pages/pdf/ eje 2/pdf 2 colombia/C025.pdf

Flores, L. E., Flores, G. A., Jiménez, R. E., Madrigal, J.C. y Perearnau, M. Á. (2009). Comunidad aprendiente. San José, Costa Rica: Ediciones Sanabria.

Hernández, A. (septiembre, 2009). Expediciones pedagógicas a las prácticas educativas exitosas en la Facultad de Estudios Superiores Zaragoza, el caso de la Licenciatura de Cirujano Dentista, en el área Biológica. Trabajo presentando en el $\mathrm{X}$ congreso nacional de investigación educativa, Veracruz, México. Recuperado de http://www.comie.org.mx/ congreso/memoriaelectronica/v10/pdf/area tematica 14/ponencias/0719-F.pdf

Lépiz, C. H. y Dengo, M. E. (1999). Moisés Vincenzi y la educación humanista. Heredia, Costa Rica: EUNA.

Luna, A. (julio, 2011). Producción colectiva de saber. Trabajo presentado en el VI Encuentro Iberoamericano de Colectivos Escolares y Redes de Maestros/as que hacen investigación e innovación desde la escuela. Argentina. Recuperado de http://www.colectivoeducadores. org.ar/cd 6to encuentro/ pages/pdf/eje 3/pdf 3 colombia/C040.pdf

Ministerio de Educación de Ciencia y Tecnología (MECT) y la Organización de los Estados Americanos Agencia Internacional para la Cooperación y el Desarrollo (OEA-AICD). (2005). La documentación narrativa de experiencias pedagógicas: Una estrategia para la formación de docentes. Buenos Aires. Recuperado de http://www.lpp-buenosaires.net/ documentacionpedagogica/ArtPon/PDF ArtPon/Doc OEA Formacion.pdf

Muñoz, V. (2005). El derecho humano a la educación: Retos y desafíos. Heredia, Costa Rica: Publicaciones Universidad Nacional.

Rodríguez, L. y Forero, N. (julio, 2011). El viaje como alternativa de formación en la expedición pedagógica. Trabajo presentado en el VI encuentro iberoamericano de colectivos que hacen investigación en la escuela, Córdoba, Argentina. Recuperado de http://www. colectivoeducadores.org.ar/cd 6to encuentro/ pages/pdf/eje 2/pdf 2 colombia/ c021x.pdf 
Suárez, H. (Ed.). (2003). Caminantes y caminos. La expedición pedagógica en Bógota. Bógota: Editora Guadalupe.

Torío, S., Peña, J. V. y Fernández, C. M. (2010). Evaluación de una experiencia de innovación docente para el aprendizaje significativo en pedagogía: Un marco para la reflexión a partir de la percepción de los estudiantes. Estudios Sobre Educación, 18, 229-253. Recuperado de http://dspace.unav.es/dspace/bitstream/10171/9827/2/ESE 18 10.pdf

Unda, M. P. (marzo, 2008). El viaje en la expedición pedagógica colombiana: Propuesta para pensar nuestro V Encuentro Iberoamericano de Redes y Colectivos de Maestros. Trabajo presentado en el Seminario preparatorio del $V$ encuentro iberoamericano realizado por el movimiento expedición pedagógica, Bogotá. Recuperado de http://www.reddhie.org.ar/paginas/ encuentros/iberoamericanos/encuentros iberoamericanos/art viaje producc saber.pdf

Whitman, W. (2012). No te detengas. [Versión de Leando Wolfson]. Recuperado de http://grego. es/?p=3844

\section{Cómo citar este artículo en APA:}

Vásquez, É. (enero-abril, 2014). Un camino hacia el cambio en el aprendizaje: La expedición como recurso pedagógico en el ámbito universitario. Revista Electrónica Educare, 18(1), 57-76. Recuperado de http://www.revistas.una.ac.cr/index.php/EDUCARE/issue/current

Nota: Para citar este artículo en otros sistemas puede consultar el hipervínculo "Como citar el artículo" en la barra derecha de nuestro sitio web:

http://www.revistas.una.ac.cr/index.php/EDUCARE/index 